Nanostructure and crystallization phenomena in multilayered films of alternating iPP and PA6 semicrystalline polymers.

F. Ania, F.J. Baltá-Calleja, A. Flores, G.H. Michler, S. Scholtyssek,

D. Khariwala, A. Hiltner, E. Baer, L. Rong, B. S. Hsiao

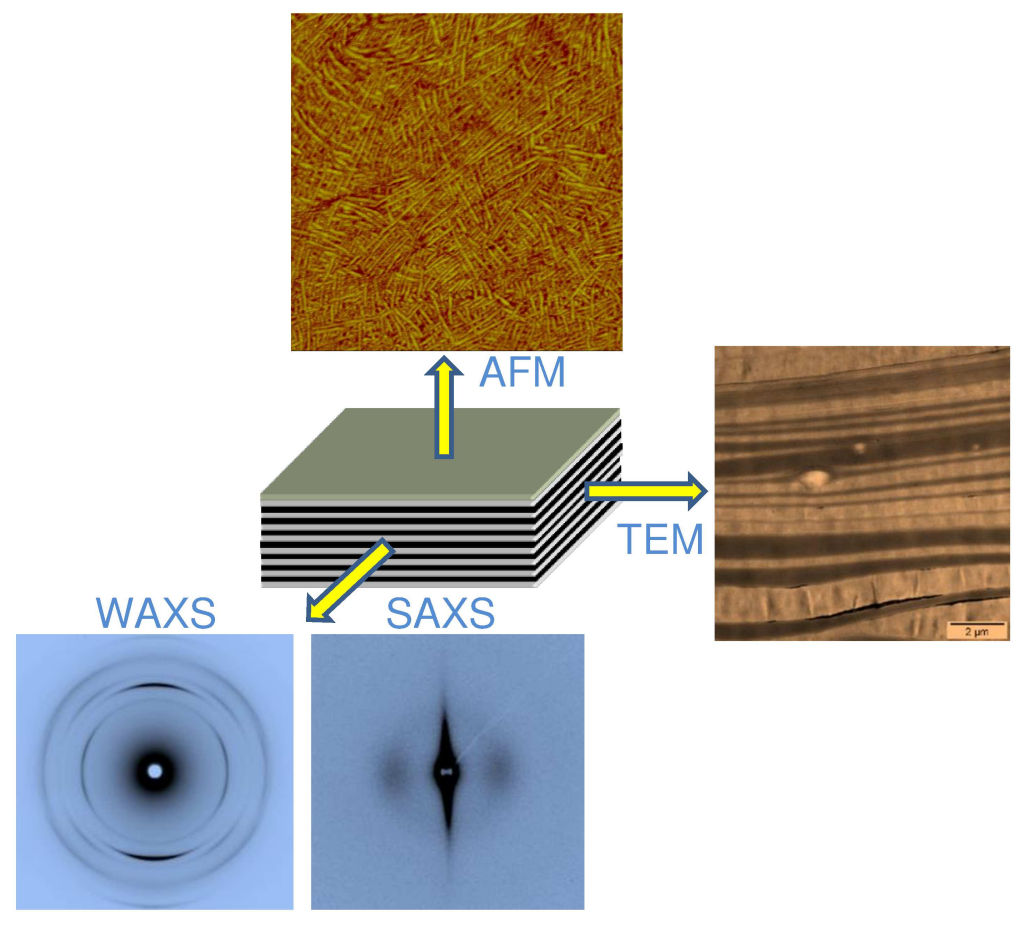




\title{
Nanostructure and crystallization phenomena in multilayered films of alternating iPP and PA6 semicrystalline polymers.
}

F. Ania ${ }^{a} *$, F.J. Baltá-Calleja ${ }^{a}$, A. Flores ${ }^{\text {a }}$, G.H. Michler ${ }^{b}$, S. Scholtyssek ${ }^{b}$, D. Khariwala ${ }^{c}$, A. Hiltner ${ }^{c}$, E. Baer ${ }^{\text {, }}$ L. Rong ${ }^{d}$, B. S. Hsiao ${ }^{d}$

${ }^{\text {a }}$ Instituto de Estructura de la Materia, IEM-CSIC, Serrano 119, E-28006 Madrid, Spain.

b Institute of Physics, Martin-Luther-University Halle-Wittenberg, D-06099 Halle (Saale), Germany.

${ }^{c}$ Department of Macromolecular Science, Case Western Reserve University, 2100 Adelbert Road, Cleveland, OH 44106-7202, USA.

${ }^{\mathrm{d}}$ Department of Chemistry, Stony Brook University, Stony Brook, NY, 117943400 , USA.

\begin{abstract}
The present work is concerned with the study of the crystalline morphology and the nanostructure of a multilayered system of two alternating immiscible semicrystalline polymers: isotactic polypropylene (iPP) and polyamide 6 (PA6). Films with a volume ratio of 70/30 were prepared by means of layer multiplying coextrusion. Contrary to previous experiments, performed with semicrystalline/amorphous and amorphous/amorphous nanolayered systems, the studied iPP/PA6 film does not exhibit a well defined maximum in the USAXS patterns. This result accounts for an irregular layered structure, as further confirmed by means of TEM images. Nevertheless, such a layered assembly still influences the crystallization behaviour of both constituent polymers. On the one hand, the crystallization of PA6 within the multilayered material is substantially hindered as evidenced by its weak scattering intensity. Real time studies as a function of temperature undoubtedly detect the presence of a WAXS peak and a SAXS maximum associated to PA6 above the melting temperature of iPP. Room temperature AFM studies also confirm the occurrence of crystalline structures within the PA6 layers. On the other hand, SAXS and WAXS measurements at room temperature reveal the occurrence of an oriented lamellar morphology within the iPP layers bearing uniaxial symmetry around an axis perpendicular to the layers surface. Results show that the crystalline molecular chains are placed mainly parallel to the layer surfaces forming edge-on lamellae. Moreover, X-ray scattering results are in agreement with the occurrence of two populations of lamellae, both edge-on and perpendicular to each other, in agreement with the crosshatched morphology observed by AFM.
\end{abstract}

\footnotetext{
* Corresponding author: emfernando@iem.cfmac.csic.es; phone: +34917459506; fax: +34915642431
} 


\section{Introduction}

Nanostructural polymer systems are known to be potential candidates to develop synergistic effects which could yield better properties than those of the pure components. In contrast to the self-assembled confinement often developed in microphase-separated block copolymers [1], layer-multiplying coextrusion uses forced assembly to produce films with hundreds or thousands of alternating layers. In this way, pairs of immiscible polymers can be fabricated into an unlimited length of nanolayered films having layers less than $10 \mathrm{~nm}$ in thickness. Although the amount of material in a single confined layer is very small, its characteristic properties are multiplied many-fold by the number of identical layers in the assembly. This allows using conventional experimental techniques to probe the effect of physical confinement on size-scale-dependent properties [2,3].

In preceding papers [4,5], the study of the forced assembly of co-extruded pairs of immiscible polymers using ultra-small-angle x-ray scattering (USAXS) shed light on several details of their highly regular nanolayered structure. The experimental long spacings found in multilayered films of a semicrystalline/amorphous system such as poly(ethylene terephthalate)/polycarbonate (PET/PC) and those for an amorphous/amorphous system such as poly(methyl methacrylate)/poly(styrene) (PMMA/PS) showed values which correlate well with the nominal periodicities of the stacks and with the layer periodicities derived, on a more local scale, from atomic force microscopy (AFM) images. The thermal stability of the laminated architecture of the films was also tested at temperatures even higher than the glass transition temperature of both constituent polymers. For the PET/PC system, the multilayers are well preserved after heat treatments up to $\mathrm{T}_{\mathrm{a}} \approx 150^{\circ} \mathrm{C}$, showing only a large increment of the USAXS scattering power due to the higher electron density difference among alternating layers provoked by the crystallization of PET [4]. The PMMA/PS system in turn, shows the absence of USAXS maxima after heat treatments at an intermediate range of temperatures $\left(110-130^{\circ} \mathrm{C}\right)$; while AFM evidences that the nanolayered structure is mostly preserved. This fact was tentatively explained in the light of spinodal dewetting processes occurring between the forced assembled polymer layers. Above $140^{\circ} \mathrm{C}$, the interfacially driven break-up of the layers ends up with the final disappearance of the multilayered PMMA/PS structure [5].

Most structural work published on multilayered polymer systems has been based on x-ray scattering and AFM experiments and only few papers have shown transmission electron microscopy (TEM) studies. However, high resolution TEM has been proved to be a very convenient technique to investigate the structure and morphology of multilayered systems. 
For instance, in case of alternating isotactic polypropylene and polystyrene (iPP/PS) layers, TEM results revealed a well developed layered structure for the volume compositions 30/70, $50 / 50$ and 70/30 [6]. On the contrary, for very uneven compositions (10/90 or 90/10), a significant number of failures in the layered structure, such as layer endings or formation of droplets, were observed. These defects become more frequent, the thinner the layer thickness is. A further example of TEM investigations deals with PET/PC multilayers that showed macroscopically aligned continuous layers of the constituent polymers having quite uniform thickness [7]. On annealing the samples, the PET layers undergo cold-crystallization and, at the end of the crystallization process, the size of the developed lamellar crystals is found to be independent of the thickness of individual layers. This result was further confirmed by means of a SAXS analysis which aimed to understand the role of finite-size effects on the development of crystalline lamellae in semicrystalline polymers [8]. It was shown that, during the isothermal crystallization of PET/PC multilayered films at $117^{\circ} \mathrm{C}$, both the induction period and crystallization rate are influenced by the degree of physical confinement of PET [8]. The former increases with decreasing PET layer thickness, while crystallization rate slows down with confinement. Nevertheless, the analysis of SAXS curves, undertaken by means of the interface distribution function (IDF) assuming a dual lamellar stack model revealed that the lamellar stacks of PET consist on an average of 3 or 4 correlated crystals and, once formed, PET crystallites are very similar and independent of the PET layer thickness of the films. Furthermore, the linear crystallinity (crystallinity within lamellar stacks), was found to be much higher than the total degree of crystallinity obtained by WAXS experiments. This suggests that not all the volume of PET is filled with stacks of crystals but these stacks are separated by large amorphous zones in agreement with earlier findings in non-confined PET. Another interesting issue concerning finite-size effects in multilayered materials is the emergence of lamellar orientation [3,9-11]. Whether edge-on or flat-on lamellae are generated within the confined layers seems to depend on the nature of the polymer materials and the conditions of crystallization. For instance for coextruded layers of polyethylene oxide (PEO) and poly(ethylene-co-acrylic acid) (EAA), confined PEO lamellae were reported to be oriented primarily parallel to the layer surface (flat-on lamellae) as the PEO layer thickness decreased below about $100 \mathrm{~nm}$ [3]. On the contrary, the confinement in multilayered films with alternating layers of a high density polyethylene (HDPE) and polystyrene (PS) resulted in a change from banded discoids of mostly edge on lamellae at the microscale to long bundles of edge-on lamellae with polymer chains parallel to the layer interface at the nanoscale [11]. For iPP/ PS films, the thin iPP layers were found to be mainly composed of edge-on lamellae with 
(040) planes lying flat on the interface [9]. However, when iPP layers were $65 \mathrm{~nm}$ thick or even thinner, an additional crystal population with (110) planes parallel to the interface and (040) planes perpendicular to the interface became evident. Finally, for the PET/PC system, it has also been proposed that two lamellar populations develop: edge-on lamellae appearing close to the interfaces and flat-on lamellae preferentially located in the layer core [10].

In the present study we wish to extend the above investigations to a new system formed by two semi-crystalline polymers such as iPP and polyamide 6 (PA6). The iPP/PA6 blends are of significant commercial interest due to a good balance of properties: PA6 shows an excellent mechanical behaviour and iPP can provide a strong resistance against moisture and ensures good processability [12]. Because of their incompatible character due to a very low mixing entropy, a variety of compatibilizers, such as grafted PP and maleated PP, have been used to reduce the interfacial tension and achieve more stable PP/PA6 systems [13]. Multilayered iPP/PA6 films can, in principle, give rise to a nanostructured assembly without the need of adding any compatibilizers.

Our aim is to investigate the morphology and the multilayered nanostructure of the iPP/PA6 system and to find out the influence of the layered arrangement on the crystalline structure of the constituent polymers, at room temperature and during thermal treatments. For this purpose, WAXS and SAXS experiments, together with TEM and AFM observations have been analyzed.

\section{Experimental Part}

\subsection{Materials}

Isotactic polypropylene P4G2Z-159 was supplied by Huntsman (USA) and polyamide 6 Ultramid B33 01 was supplied by BASF. The main characteristics of both materials, as supplied in the manufacturers' data sheets are collected in Table 1. The iPP/PA6 multilayered films were produced on a laboratory scale coextrusion line at Case Western Reserve University that incorporates layer-multiplying technology [14]. The system, consisting of 512 alternating layers, has an iPP/PA6 volume ratio of 70/30. Films were produced in a continuous manner with approximately $20 \mathrm{~cm}$ width and $100 \mu \mathrm{m}$ thickness. The resulting thickness fluctuates across the films between 50 and $150 \mu \mathrm{m}$. According to this, the expected nominal iPP layer thickness ranges from $150 \mathrm{~nm}$ to $400 \mathrm{~nm}$ and from 50 to $200 \mathrm{~nm}$ for PA6. Control films, coextruded in the same manner, but using one at a time of the two polymers (iPP or PA6) were also produced for the sake of comparison. 


\subsection{X-ray scattering}

Small (SAXS) and wide angle X-ray scattering (WAXS) measurements were performed at the beamline X27C of the National Synchrotron Light Source (NSLS) at Brookhaven National Laboratory, USA. The x-ray wavelength was fixed to $0.1371 \mathrm{~nm}$. The sample-detector distance was calibrated by means of standards with known sharp reflections. Silver behenate and aluminum trioxide were used to derive sample-detector distances of $2060 \mathrm{~mm}$ and $127 \mathrm{~mm}$ for SAXS and WAXS, respectively. The scattered intensity was recorded using a two-dimensional (2D) MARCCD detector in 1024 x 1024 pixel arrays. The size of each pixel was $158 \mu \mathrm{m}$, both in horizontal and vertical directions.

At room temperature, the incident beam was directed along the three orthogonal directions of the specimens. For experiments as a function of temperature, polymer films were placed with their surfaces perpendicular to the x-ray beam inside a hot-stage. Background subtraction and data analysis (projections, averaging...) were accomplished by means of the Fit-2d program [15].

USAXS results were obtained at HASYLAB, in the BW4 beamline. The sample-detector distance was approximately $13 \mathrm{~m}$, giving spatial resolution limits between 23 and $650 \mathrm{~nm}$ [16]. The x-ray wavelength $\lambda$ was fixed to $0.138 \mathrm{~nm}$ and the data were recorded using a MARCCD 165 camera with a resolution of $2048 \times 2048$ pixels ( $79 \mu \mathrm{m}$ per pixel).

\subsection{Microscopic techniques}

For the morphology investigation different microscopic techniques were used. The layer morphology was studied by means of a transmission electron microscope (TEM, LEO 912, Germany) with an accelerating voltage of $120 \mathrm{kV}$. The iPP/PA6 films were embedded into epoxy resin, cross sectioned and afterwards stained with ruthenium tetroxide $\left(\mathrm{RuO}_{4}\right)$ vapour for several hours or with osmium tetroxide $\left(\mathrm{OsO}_{4}\right)$ formalin solution for at least one week. Both staining techniques were done at $60^{\circ} \mathrm{C}$. Subsequently, ultra-thin sections ( $80 \mathrm{~nm}$ thick) were ultramicrotomed with a diamond knife at room temperature.

The structure of the layer surfaces was investigated on delaminated films using atomic force microscopy (AFM). The AFM (multimode with the controller NanoScope IIIa) was used in the tapping mode and the samples were scanned under moderate tapping conditions (set point ratio $=0.9)$ using microfabricated silicon cantilevers. Figure 1 sketches the extraction of the samples 
from the films: a) TEM sections for the morphology investigation and b) AFM samples for the investigation of the layer surfaces.

\section{Results and Discussion}

\subsection{Morphology}

Figure 2 illustrates some particular features found by TEM in the nanolayered iPP/PA6 films. Although iPP and PA6 layers can be clearly distinguished in both images, it is apparent that the thickness of the layers is very irregular. Ending and merging of layers is a frequently found morphological characteristic. In addition to layered regions, other distinct areas without such morphology, but rather having the character of a particle-matrix structure, can be found. The origin of the poorly developed layered structure in the iPP/PA6 system, as compared for instance with the good layer uniformity found in the iPP/PS one [9], could be due to viscosity differences between the polymers in the layers and/or elastic effects that introduce secondary flows [17].

Due to the different staining procedure, iPP appears bright in Figure 2a and dark in Figure $2 \mathrm{~b}$. Staining with $\mathrm{OsO}_{4}$-Formalin (Fig. 2a) was expected to reveal the morphology of PA6, whereas a treatment with $\mathrm{RuO}_{4}$ (Fig. 2b) should favour the visualization of the inner structure of iPP. However, neither the crystalline morphology of PA6, nor that of iPP could be distinguished in the TEM micrographs.

AFM investigations were carried out in order to provide additional evidence of whether crystalline lamellae develop in this multilayered material. For this purpose the films were delaminated and AFM images were taken on both generated surfaces after delamination (Figs. 3 and 4). For the iPP surface, the height image (Fig. 3a) offers no information about the iPP crystalline morphology. On the contrary, the phase image (Fig. 3b) shows a well defined typical cross-hatched crystalline structure, with no clear evidence of a higher-level spherulitic organization [18]. On the other hand, both phase and height AFM images on the PA6 surface (Fig. 4a and b) show a very clear spherulitic morphology. Results suggest that both polymers exhibit crystalline lamellar structures, even though TEM did not reveal similar crystalline morphologies on the surfaces perpendicular to those shown in Figures 3 and 4 .

\subsection{X-ray scattering}

\subsubsection{USAXS results}


The USAXS curves of the iPP/PA6 system with the layers parallel to the x-ray beam do not show any maximum at room temperature. Although the difference in electron density between iPP and PA6 is not very large, other similar systems do show the presence of several scattering maxima [4,5]. It is then concluded that, in this case, the absence of USAXS peaks should be related to the irregular layered structure evidenced in the TEM micrographs (Fig. 2).

\subsubsection{WAXS and SAXS results at room temperature}

Room temperature WAXS 2D-patterns of the control films are presented in Fig. 5. Each film has been irradiated in two perpendicular directions. Figures $(5 \mathrm{a}, 5 \mathrm{~b})$ and $(5 \mathrm{c}, 5 \mathrm{~d})$ correspond to iPP and PA6, respectively. In all cases, practically isotropic diffraction rings, multiple reflections for iPP and a broad double reflection for PA6, can be observed. Similarly, the SAXS patterns of the iPP control film show an isotropic maximum in both directions (Fig. 6a and b). For the parallel configuration (x-ray beam along MD), a quite strong streak along the meridian, which is the direction of uniaxial symmetry perpendicular to the layer surfaces (ND), can also be observed (Fig. 6b). A similar streak has been reported before for the PP control material of an iPP/PS series of multilayered films and it was attributed to scattering from the interfaces, denoting an incomplete fusion of the iPP layers during the coextrusion process [9]. Finally, the PA6 control film also presents typical non-oriented SAXS patterns, but with such a low intensity contrast, that it is difficult to perceive the maximum without a proper integration. In the case of the x-ray beam being directed parallel to the film surfaces, a meridional streak is also present.

Fig. 7 summarizes the initial WAXS (a, b) and SAXS (c, d) 2D-patterns at room temperature of the iPP/PA6 system with the x-ray beam along ND (a, c) and MD (b, d). Both WAXS and SAXS images obtained perpendicularly to the surfaces (a, c), exhibit quasi-isotropic scattering rings, with a slight preferential orientation along the extrusion direction. The parallel configuration, in turn, reveals a well developed orientation which will be discussed in detail below. For this setup, x-ray diagrams look identical either taken along MD or TD (the latter not shown in the figure).

Linear WAXS and SAXS curves as a function of the diffraction angle $2 \theta$, obtained from Figs 5, 6 and 7 are shown in Figure 8. Figure 8a shows the WAXS curves derived by azimuthally averaging the isotropic 2D-patterns of the control films and the same procedure has been applied to the oriented patterns of the 70/30 iPP/PA6 film, taken with the x-ray beam parallel (MD) and perpendicular (ND) to the layers surface. Azimuthal averaging means that for each 
chosen distance from the centre of the pattern, the intensity of all the pixels on the circular ring is summed and divided by the number of pixels giving thus rise to an averaged scattering curve [19]. Such integration is only valid for isotropic patterns. For the oriented patterns of iPP/PA6, the same procedure has been used with the sole purpose of comparing the angular position of the observed reflections with those of the control materials. One should be cautious about the fact that some reflections, especially around the meridian, may not appear due to orientation. By means of a peak fitting analysis, the experimental curves of the neat iPP and PA6 control materials have been deconvoluted into a constant baseline, amorphous halo (represented by a gaussian) and separate crystalline peaks (represented by Pearson VII curves). The crystallinity index for iPP and PA6 control materials has been estimated to be around 60 and 50\%, respectively. Comparing the results of the iPP control material with the well established unit cell parameters of the monoclinic $\alpha$-modification of iPP [20], it is found that only crystalline reflections of the $\alpha$-form are present. With increasing diffraction angle, the most intense ones can be indexed as: (110), (040), (130), (111) and (-131). The WAXS curve for the PA6 control film exhibits two clearly defined broad peaks centred at around $2 \theta=21.5^{\circ}\left(\gamma_{1}(001)\right)$ and $22.8^{\circ}$ $\left(\gamma_{2}(200)\right)$ and two other weaker peaks at around $2 \theta=20.2^{\circ}\left(\alpha_{1}(200)\right)$ and $23.9^{\circ}$ $\left(\alpha_{2}(002 / 202)\right)$ [21]. This means that there is a minor content $(\sim 35 \%)$ of the most thermodynamically stable $\alpha$-crystal modification composed of extended nylon 6 chains. Most of the material shows instead $\gamma$-form crystals, which are known to be composed of pleated chains with a hexagonal or pseudohexagonal packing. Both modifications have been sometimes found to coexist in various percentages depending on processing conditions. It is generally accepted that rapid cooling favors the $\gamma$-form crystallization. The monoclinic $\alpha$-form is the preferred structure in solution-cast and annealed nylon 6 samples, while the $\gamma$-crystalline form seems to predominate in melt-spun nylon 6 fibers, nylon 6/clay nanocomposites and electrospun nylon 6 nanofibers [22].

The angular position of the WAXS peaks of the co-extruded film, parallel and perpendicular to the $\mathrm{x}$-ray beam maintain the crystalline reflections of the iPP $\alpha$-form. However, their relative intensity as shown in the patterns of Fig. 7 ( $a$ and b) is drastically changed. It is clearly observed that the (040) and the (130) peaks appearing, respectively, at $2 \theta=16.9^{\circ}$ and $18.7^{\circ}$ practically disappear when the $\mathrm{x}$-ray beam perpendicularly illuminates the film. This accounts for the fact that the (040) planes are predominantly adopting an orientation parallel to the layers interface [9]. On the other hand, no reflections of the $\gamma$-modification of PA6 seem to be present in the WAXS curves of the iPP/PA6 sample. Although their presence cannot be 
completely ruled out due to the fact that the strongest $\gamma$-peak $\left(2 \theta_{001} \sim 21.5^{\circ}\right)$ of such crystals practically coincides with the (111) and (-131) reflections of iPP, results suggest that PA6 crystallites in the multilayered film preferentially adopt the $\alpha$-form. This can be inferred by the presence of two weak peaks at $2 \theta=20.2^{\circ}$ and $23.9^{\circ}$, appearing for both investigated directions, which should correspond to the (200) and (002/202) reflections of the monoclinic $\alpha$ modification [21].

At this point, it is worthy to remark the low diffracted intensity arising from the PA6 layers. It is clear that PA6 is the minor component in our 70/30 system and this proportion must be reflected in the diffracted contribution of the iPP/PA6 film. However, even in the pure PA6 control film the obtained intensity is still poorly defined. The origin could arise in both cases from spatial confinement within the PA6 domains which could restrict segmental motions. It was mentioned above that a strong SAXS meridional streak associated to scattering from the interfaces due to imperfect fusion of the layers can be observed in both the control material and the multilayered one. It is known $[23,24]$ that such confinement can produce significant crystallization supercoolings due to the fact that active heterogeneities can become restricted to a relatively small fraction of the material. For rapidly cooled samples, as it is the case of coextruded multilayers, such a supercooling may result in a hindered crystallization process where only very small and/or imperfect PA6 crystals appear. This fact is also supported by the broad WAXS crystalline peaks observed.

Fig. $8 \mathrm{~b}$ collects the SAXS curves, as a function of the scattering vector $\mathrm{q}$, derived from the 2Dpatterns shown in Figs. 6 and 7 and also from the one corresponding to the control film of PA6. For isotropic patterns (control films and iPP/PA6 in the perpendicular arrangement), the onedimensional SAXS intensity was derived by azimuthally averaging over $360^{\circ}$ and then applying the Lorentz correction. For iPP/PA6 with x-ray beam along MD, the oriented scattering was projected in reciprocal space on the plane perpendicular to the symmetry axis $\left(s_{3}\right)$ according to [19]:

$$
\{I\}_{2}\left(s_{12}\right)=2 \int_{0}^{\infty} I\left(s_{12}, s_{3}\right) d s_{3}
$$

In this case, $s_{3}$ is parallel to ND in real space and the projection, although a plane, can be represented by a curve because such a plane shows 2D-isotropy. The obtained SAXS curve for the iPP/PA6 film contains a single broad scattering maximum. The broad peak reflects the 
distribution of long spacings within the transversal plane, with a limiting higher value corresponding to the long spacing of stacks perpendicular to the x-ray beam and diminishing thereafter, in size and intensity, for other possible orientations. The maximum corresponds to a long spacing $L$ of approximately $14.5 \mathrm{~nm}(\mathrm{~L}=2 \pi / \mathrm{q})$ which is larger than the one found for the control film of iPP ( 12.5 nm). Larger long spacing values for reduced layer thickness have been previously reported for other crystallisable materials within similar multilayered assemblies [10]. The result was attributed to crystals being separated by larger amorphous regions. At the position where the SAXS peak corresponding to the polyamide PA6 (L 6.0 $\mathrm{nm}$ in the PA6 control material) should appear, there is no clear indication of any scattering peak. On the contrary, for the iPP/PA6 sample in the perpendicular arrangement the presence of two scattering maxima is evident. The calculated long spacings are 15.3 and $6.7 \mathrm{~nm}$ suggesting that they reflect the average distance among stacks of iPP and PA6 crystals, respectively. However, one must be cautious about the fact that the lower periodicity only becomes apparent after applying the Lorentz correction to the isotropic scattering and it has been reported that such procedure can produce artificial Bragg periodicities [25].

On the other hand, it can be recalled that the AFM images of the iPP and the PA6 surfaces show, in both cases, crystalline morphologies (Figs. 3 and 4). Moreover, DSC measurements of the investigated iPP/PA6 multilayered film have shown two strong endothermic peaks at $161^{\circ} \mathrm{C}$ and $219^{\circ} \mathrm{C}$ upon heating. Each peak is related to the melting process of the two pure components of the system. This result suggests that PA6 layers exhibit a significant degree of crystallinity, although it could be a consequence of a crystalline reorganization during the heating cycle which ends up with a clear transition into the liquid state. It is then interesting to investigate with more detail whether both crystalline phases can be undoubtedly detected by following their evolution in a heating and cooling cycle using x-ray diffraction.

\subsection{Temperature changes and recrystallization}

Real time WAXS and SAXS experiments with the x-ray beam along ND have been performed as a function of temperature. Isotropic 2D-patterns were found in all cases, in agreement with the room temperature results of Figs. 7a and 7c. WAXS and SAXS curves were obtained by azimuthally averaging the consecutive patterns.

Fig. 9 collects WAXS curves of an iPP/PA6 (70/30) film as a function of increasing temperature at $5^{\circ} \mathrm{C} / \mathrm{min}$ up to $200^{\circ} \mathrm{C}$. At the beginning of the experiment, at room temperature, it is observed the already described diffraction curve (Fig. 8a) with the typical reflections of the 
$\alpha$-modification of iPP and the weak traces of PA6. On increasing the temperature above the melting point of $\mathrm{iPP}\left(\mathrm{T}_{\mathrm{m}} \approx 165^{\circ} \mathrm{C}\right)$, its characteristic peaks disappear and the only crystalline peak left effectively corresponds to high temperature (HT) hexagonal form of the PA6 [21]. On cooling back, iPP and PA6 crystallize again into their respective $\alpha$-forms.

Selected SAXS curves at different increasing temperatures of the heating cycle are displayed in Fig. 10. It can be observed that the main scattering maximum associated to the iPP lamellae shifts to lower diffraction angles upon heating. It is to be noted that on melting iPP, above $165^{\circ} \mathrm{C}$, a small maximum at $\mathrm{q} \sim 0.8 \mathrm{~nm}^{-1}$ still remains (Fig. 10, insert). This maximum in fact can be weakly detected from the first room temperature diagram and it can be definitively associated to PA6 lamellae. The analysis of all the Lorentz corrected SAXS data permits to derive the long spacings arising from the crystalline-amorphous nanostructure within the layers. These long spacings are collected in Fig. 11 as a function of temperature during a heating cycle similar to that of Fig. 9, followed by a holding time of 4 minutes at $200^{\circ} \mathrm{C}$ and then cooling back to room temperature at $5^{\circ} \mathrm{C} / \mathrm{min}$. The SAXS maximum corresponding to the iPP multilayers increases from $\mathrm{L}=15.3 \mathrm{~nm}$ at room temperature to more than $30 \mathrm{~nm}$ just before melting. The noticeable variation of L close to the melting point has been frequently observed, not only in PP but also in many other polymers, and is associated to the sequential melting of crystalline lamellae giving rise to a larger periodicity between the remaining crystallites [26]. During the cooling cycle the iPP long period reappears at around $140^{\circ} \mathrm{C}$ with a value of $17.9 \mathrm{~nm}$ and decreases to $17.1 \mathrm{~nm}$ at room temperature. The PA6 long period in turn does not vary so much. It starts with a value of $6.7 \mathrm{~nm}$, increases up to $7.3 \mathrm{~nm}$ at the highest temperature and then, on cooling, grows again to yield a final long period of $7.7 \mathrm{~nm}$ back to ambient temperature.

Most interesting is the thermal stability shown by the multilayered film after having been melted one of its two components. The PA6 solid domains act as a template for the molten iPP, keeping the film macroscopically undamaged at least for a short period of time.

\subsection{Orientation in the iPP layers}

It has already been shown that the crystal arrays within the control materials do not present any preferred orientation. Regarding the PA6 contribution in the iPP/PA6 film, it is difficult to draw any conclusion due to its low scattering intensity, although the available data seem to discard any traces of molecular or crystalline stack orientation. On the contrary, the iPP contained in the same material yields well oriented WAXS and SAXS patterns (Figs. 7b and 
7d) when layer surfaces are parallel to x-rays. The (110) WAXS reflection exhibits a clear double maximum centred on the equator. In addition, the maximum of intensity of the strong (040) reflection appears on the meridian. Taking into account that its intensity becomes very weak when the film is illuminated perpendicularly (Figure 7a), it must be implied that the (040) planes are predominantly parallel to the layer surfaces. Figure $7 \mathrm{a}$ also reveals that the lamellae do not have any clear preferred orientation when looking at the film along ND. Moreover, WAXS (and SAXS) patterns are identical when the beam is applied either along the extrusion direction or perpendicular to it, confirming that the lamellar arrangement exhibits uniaxial symmetry around an axis perpendicular to the layers surface. Considering all the obtained information, results suggest that iPP crystals are oriented with their $\mathrm{c}$ and $\mathrm{a}^{*}$-axis $\left(\mathrm{a}^{*}\right.$ is the reciprocal a-axis) lying in the plane of the layer stacks, b being the axis of uniaxial symmetry. This means that iPP crystalline molecular chains are placed mainly parallel to the layer surfaces, forming edge-on lamellae. From the point of view of SAXS, these lamellae are responsible for the well oriented maxima observed in Fig. 7d and the isotropic ring of Fig. 7c. The corresponding long period $(\mathrm{L} \sim 15 \mathrm{~nm})$ is in good agreement with those found for other iPP samples [26]. Preceding studies suggest that lamellar branching manifests itself in nearly every crystallization condition of the $\alpha$-form of iPP [27-30]. Our WAXS results do not reveal specific maxima associated to daughter lamellae. However, according to the literature, branching should generate at approximately right angles to the parent ones. In our case, the c and $\mathrm{a}^{*}$-axis of the secondary lamellae should also lie in the plane of the layer interfaces, $\mathrm{b}$ still being the axis of uniaxial symmetry. Hence, these second edge-on lamellar population would not produce additional WAXS or SAXS reflections other than those of the parent lamellae. The crosshatched lamellar morphology shown in the TEM image of Figure 3 can thus be explained by the two types of populations above described, both edge-on and practically perpendicular to each other. The uniaxial symmetry, perpendicular to the film surfaces, exhibited by the crystal arrangement in the iPP layers could be compatible with a discoid morphology (mesh texture shown in Figure 3) arising from the limited thickness of the individual layers, as already proposed for similar multilayered films of iPP/PS [9] or HDPE/PS [11].

Figure 3 also suggests that the orthogonally grown lamellae exhibit similar crystal thicknesses than the parent ones. This result is in agreement with previous findings suggesting that large supercoolings promote arrays of lamellae with little structural differences [31 and references therein]. 
The isotropic WAXS and SAXS rings found for the control film of iPP (Figs. 5 and 6 , respectively) clearly demonstrate that the flow generated during the extrusion process is not able to induce any kind of orientation. For the iPP/PA6 film, it has already been mentioned that, at room temperature, the crosshatched lamellar morphology is also randomly oriented in any plane parallel to the interfaces. However, SAXS experiments as a function of temperature have revealed a tendency towards a four-point diagram when approaching the melting temperature of iPP. At such a point, two intensity maxima on the meridian and on the equator are observed. It could be assumed that these high melt temperature oriented lamellae were formed at the early stages of crystallization during the extrusion process and they would reflect that flow could induce an incipient preferential orientation within the plane of the layers. Such initial orientation, in flow direction and perpendicular to it, is then rapidly masked by the subsequent crystallization of unoriented lamellae organized in the form of discoids.

\section{Conclusions}

The morphology of the iPP/PA6 system was found by TEM to preserve the parallelism among the layers, but the layer thickness is not uniform across the sample. A certain number of failures, such as endings or merging of layers, give rise to isolated areas having the character of a particle-matrix structure. As a consequence, no USAXS maxima corresponding to the periodicity of the iPP/PA6 nanolayers are observed.

AFM studies on delaminated films of the multilayered material reveal a cross-hatched lamellar structure in the iPP surface at the submicron scale, while a spherulitic morphology is detected for PA6.

For the minor component PA6, room temperature WAXS experiments reveal broad maxima associated to the presence of small crystallites. Similar room temperature SAXS experiments suggest that spatial confinement occurring within the PA6 layers hinders the crystallization process.

Using real time WAXS and SAXS methods as a function of temperature, a crystalline reflection associated to PA6 and a weak peak associated to the PA6 lamellar arrangement were undoubtedly identified above the melting temperature of iPP. Results suggest that PA6 crystals adopt a $\alpha$-modification within the layered material in contrast to the mixture of $\gamma$-and $\alpha$-phases found in the control PA6 sample.

For the iPP layers, $\alpha$-form crystals are located edge-on with respect to the interfaces with their $\mathrm{c}$-axis lying in the plane of the layers and the b-axis perpendicular to it, which is consistent with an additional edge-on lamellar population appearing at right angles with respect to the 
parent lamellae. X-ray scattering results are, thus, in agreement with the crosshatched morphology observed by TEM.

It has been suggested that the extrusion flow could also play a minor role on the development of orientation within planes parallel to the interfaces during the first stages of crystallization. Finally, it is noteworthy the thermal stability shown by the multilayered film after the melting of one of its two components. The PA6 solid domains act as a template for the molten iPP, keeping the film macroscopically undamaged at least for a short period of time.

\section{Acknowledgements}

The authors gratefully acknowledge MICINN, Spain (grants FIS2007-60534 and FIS201018069), USA NSF Science and Technology Center for Layered Polymeric Systems (Grant 0423914), German Research Foundation (DFG) and European Community contract RII3-CT2004-506008 (IA-SFS), DESY project II-20070031, for generous financial support. S.S. acknowledges the Max-Buchner-Forschungsstiftung for the Research Scholarship. F.J.B. also thanks the Alexander von Humboldt Foundation for financial support during his research stay at the Institute for Technical and Macromolecular Chemistry, Hamburg University, Germany. 


\section{References}

[1] Kim SH, Misner MJ, Russell TP. Controlling orientation and order in block copolymer thin films. Adv Mater 2008; 20(24): 4851-4858.

[2] Liu RYF, Ranade AP, Wang HP, Bernal-Lara TE, Hiltner A, Baer E. Forced assembly of polymer nanolayers thinner than the interphase. Macromolecules 2005; 38(26): 1072110727.

[3] Wang H, Keum JK, Hiltner A, Baer E, Freeman B, Rozanski A, Galeski A. Confined crystallization of polyethylene oxide in nanolayer assemblies. Science 2009; 323 (5915): 757-760.

[4] Ania F, Puente Orench I, Baltá-Calleja FJ, Roth S, Khariwala D, Hiltner A, Baer E. Ultra-small-angle $\mathrm{x}$-ray scattering study of PET/PC nanolayers and comparison to AFM results. Macromol Chem Phys 2008; 209(13): 1367-1373.

[5] Ania F, Baltá-Calleja FJ, Henning S, Khariwala D, Hiltner A, Baer E. Study of the multilayered nanostructure and thermal stability of PMMA/PS amorphous films. Polymer 2010; 51(8): 1805-1811.

[6] Scholtyssek S, Adhikari R, Seydewitz V, Michler GH, Baer E, Hiltner A. Evaluation of morphology and deformation micromechanisms in multilayered PP/PS films: An electron microscopy study. Macromol Symp 2010; 294(1): 33-44.

[7] Adhikari R, Lebek W, Godehardt R, Henning S, Michler GH, Baer E, Hiltner A. Investigating morphology and deformation behaviour of multilayered PC/PET composites. Polymers for Advanced Technologies 2005; 16(2-3): 95-101.

[8] Puente Orench I, Stribeck N, Ania F, Baer E, Hiltner A, Baltá-Calleja FJ. SAXS study on the crystallization of PET under physical confinement in PET/PC multilayered films. Polymer 2009; 50(12): 2680-2687.

[9] Jin Y, Rogunova M, Hiltner A, Baer E, Nowacki R, Galeski A, Piorkowska E. Structure of polypropylene crystallized in confined nanolayers. J. Polymer Sci.: Part B: Polymer Physics 2004; 42(18): 3380-3396.

[10] Flores A, Arribas C, Fauth F, Khariwala D, Hiltner A, Baer E, Baltá-Calleja FJ, Ania F. Finite size effects in multilayered polymer systems: development of PET lamellae under physical confinement. Polymer 2010; 51(20): 4530-4539.

[11] Bernal-Lara TE, Liu RYF, Hiltner A, Baer E. Structure and thermal stability of polyethylene nanolayers. Polymer 2005; 46(9): 3043-3055. 
[12] Zhang L, Wan C, Zhang Y. Investigation on the multiwalled carbon nanotubes reinforced polyamide 6/polypropylene composites Polym Eng Sci 2009; 49(10): 1909-1917.

[13] Marco C, Ellis G, Gómez MA, Fatou JG, Arribas JM, Campoy I, Fontecha A. Rheological properties, crystallization, and morphology of compatibilized blends of isotactic polypropylene and polyamide. J Appl Polymer Sci 1997; 65(13): 2665-2677.

[14] Baer E, Kerns J, Hiltner A. Structure development during polymer processing. In: Cunha A, Fakirov S, editors. Dordrecht: Kluwer Academic Publishers, 2000. p. 327-344.

[15] Hammersley A. http://www.esrf.eu/computing/scientific/FIT2D/

[16] Roth SV, Döhrmann R, Dommach M, Kuhlmann M, Kröger I, Gehrke R, Walter H, Schroer C, Lengeler B, Müller-Buschbaum P. Small-angle options of the upgraded ultrasmall-angle x-ray scattering beamline BW4 at HASYLAB. Rev Sci Instrum 2006; 77(8), 085106.

[17] J. Dooley, K.S. Hyun, K Hughes. An experimental study on the effect of polymer viscoelasticity on layer rearrangement in coextruded structures. Polym Eng Sci 1998; 38(7): 1060-1071.

[18] Schönherr H, Snétivy D, Vansco GJ. A nanoscopic view at the spherulitic morphology of isotactic polypropylene by atomic force microscopy. Polym Bull 1993; 30(5): 567-574.

[19] Stribeck N. X-ray Scattering of Soft Matter. In: Springer Laboratory Manuals in Polymer Science. Springer-Verlag, 2007. p. 95-139.

[20] Lotz B, Wittmann JC, Lovinger AJ. Structure and morphology of poly(propylenes): a molecular analysis. Polymer 1996; 37(22): 4979-4992.

[21] Ibanes C, de Boissieu M, David L, Seguela R. High temperature behaviour of the crystalline phases in unfilled and clay-filled nylon 6 fibers. Polymer 2006; 47(14): 50715079 .

[22] Liu Y, Cui L, Guan F, Gao Y, Hedin NE, Zhu L, Fong H. Crystalline Morphology and Polymorphic Phase Transitions in Electrospun Nylon 6 Nanofibers. Macromolecules 2007; 40(17): 6283-6290.

[23] Tol RT, Mathot VBF, Groeninckx G. Confined crystallization phenomena in immiscible polymer blends with dispersed micro and nanometer sized PA6 droplets, part 1: uncompatibilized PS/PA6, (PPE/PS)/PA6 and PPE/PA6 blends. Polymer 2005; 46(2): 369-382.

[24] Tol RT, Minakov AA, Adamovsky SA, Mathot VBF, Schick C. Metastability of polymer crystallites formed at low temperature studied by ultra fast calorimetry: Polyamide 6 confined in sub-micrometer droplets vs. bulk PA6. Polymer 2006; 47(6): 2172-2178. 
[25] Cser F. About the Lorentz correction used in the interpretation of small angle x-ray scattering data of semicrystalline polymers. J Appl Polym Sci 2001; 80(12): 2300-2308.

[26] Ryan AJ, Stanford JL, Bras W, Nye TMW. A synchrotron x-ray study of melting and recrystallization in isotactic polypropylene. Polymer 1997; 38(4): 759-768.

[27] Lotz B, Wittmann JC. The molecular origin of lamellar branching in the $\alpha$ (monoclinic) form of isotactic polypropylene. J Polym Sci: B: Polym Phys 1986; 24: 1541-1558.

[28] Fujiyama M, Wakino T, Kawasaki Y. Structure of skin layer in injection-molded polypropylene. J App Polym Sci 1988; 35(1): 29-49.

[29] Zhu P-W, Edward G. Orientational distribution of parent-daughter structure of isotactic polypropylene: a study using simultaneous synchrotron WAXS and SAXS. J Mater Sci 2008; 43(19): 6459-6467.

[30] Varga J. Crystallization, melting and supermolecular structure of isotactic polypropylene, in: Polypropylene. Structure, blends and composites, vol. 1: Structure and Morphology, p. 57-115. Ed. Karger-Kocsis J. Chapman \& Hall, London, 1995.

[31] Dai PS, Cebe P, Capel M, Alamo RG, Mandelkern L. In situ wide- and small- angle Xray scattering study of melting kinetics of isotactic poly(propylene). Macromolecules 2003; 36(11): 4042-4050. 


\section{Figure Captions}

Fig. 1 Scheme showing the extraction methods of a) TEM sections for investigation of morphology and b) AFM samples for investigation of layer surfaces.

Fig. 2 TEM micrographs of an iPP/PA6 (70/30) nanolayered film: a) $\mathrm{OsO}_{4}$ staining and b) $\mathrm{RuO}_{4}$ staining.

Fig. 3 AFM image of the delaminated iPP surface of an iPP/PA6 film: a) height image, b) phase image.

Fig. 4 AFM image of the delaminated PA6 surface of an iPP/PA6 film: a) height image, b) phase image.

Fig. 5 WAXS patterns with $x$-ray beam along ND (left) and MD (right) for the control films of iPP $(a, b)$ and PA6 (c,d). XR = x-ray beam; MD = extrusion direction; ND =normal direction and $\mathrm{TD}=$ transverse direction.

Fig. 6 SAXS patterns with X-ray beam perpendicular (a) and parallel (b) to layer surfaces for the control film of iPP.

Fig. 7 WAXS (a, b) and SAXS (c, d) 2D-patterns of an iPP/PA6 (70/30) film showing room temperature orientation with x-ray beam along ND (left) and MD (right). Samples setup as in Figure 5. The main iPP crystalline reflections of the $\alpha$-form are signalized.

Fig. 8 a) 1D-curves calculated from the 2D-WAXS patterns of an iPP/PA6 film with x-ray beam parallel (MD) and perpendicular (ND) to layers surface. Curves of the control films of iPP and PA6 are also displayed for comparison. b) Idem for SAXS.

Fig. 9 WAXS curves of an iPP/PA6 (70/30) film as a function of increasing temperature (x-ray beam perpendicular). Main crystal reflections corresponding to iPP and PA6 are indicated.

Fig. 10 Selected SAXS patterns of a iPP/PA6 (70/30) film at different temperatures: a) $30^{\circ} \mathrm{C}$, b) $130^{\circ} \mathrm{C}$, c) $150^{\circ} \mathrm{C}$, d) $160^{\circ} \mathrm{C}$, e) $170^{\circ} \mathrm{C}$, f) $180^{\circ} \mathrm{C}$ and g) $190^{\circ} \mathrm{C}$. X-ray beam is perpendicular to the film. Insert shows a magnification of the higher q region where the PA6 maximum appears.

Fig. 11SAXS long spacings of an iPP/PA6 (70/30) film during the heating and cooling cycle up to $200^{\circ} \mathrm{C}$ of Fig. 7: ( $)$ iPP and ( $\left.\mathbf{\nabla}\right)$ PA6. 
Table 1: Properties of the constituent materials

Polypropylene P4G2Z-159

Polyamide 6 Ultramid B33 01

Melt flow rate $1.95 \mathrm{~g} / 10 \mathrm{~min}$ (ASTM-D1238)

Relative viscosity

$3.3 \quad(\mathrm{ISO}-307)$

(1\% in $96 \%$ Sulphuric acid)

Density $\quad 0.90 \mathrm{~g} / \mathrm{cm}^{3} \quad\left(\right.$ ASTM-D1505) $1.13 \mathrm{~g} / \mathrm{cm}^{3} \quad$ (ISO-1183)

Tensile yield strength $\quad 37 \mathrm{MPa}$ (ASTM-D638)

Tensile yield elongation $\quad 9 \%$ (ASTM-D638)

\begin{tabular}{lllll}
\hline Melting point & $161^{\circ} \mathrm{C}$ & (DSC, our lab) & $220^{\circ} \mathrm{C}$ & (ISO-3146)
\end{tabular} 

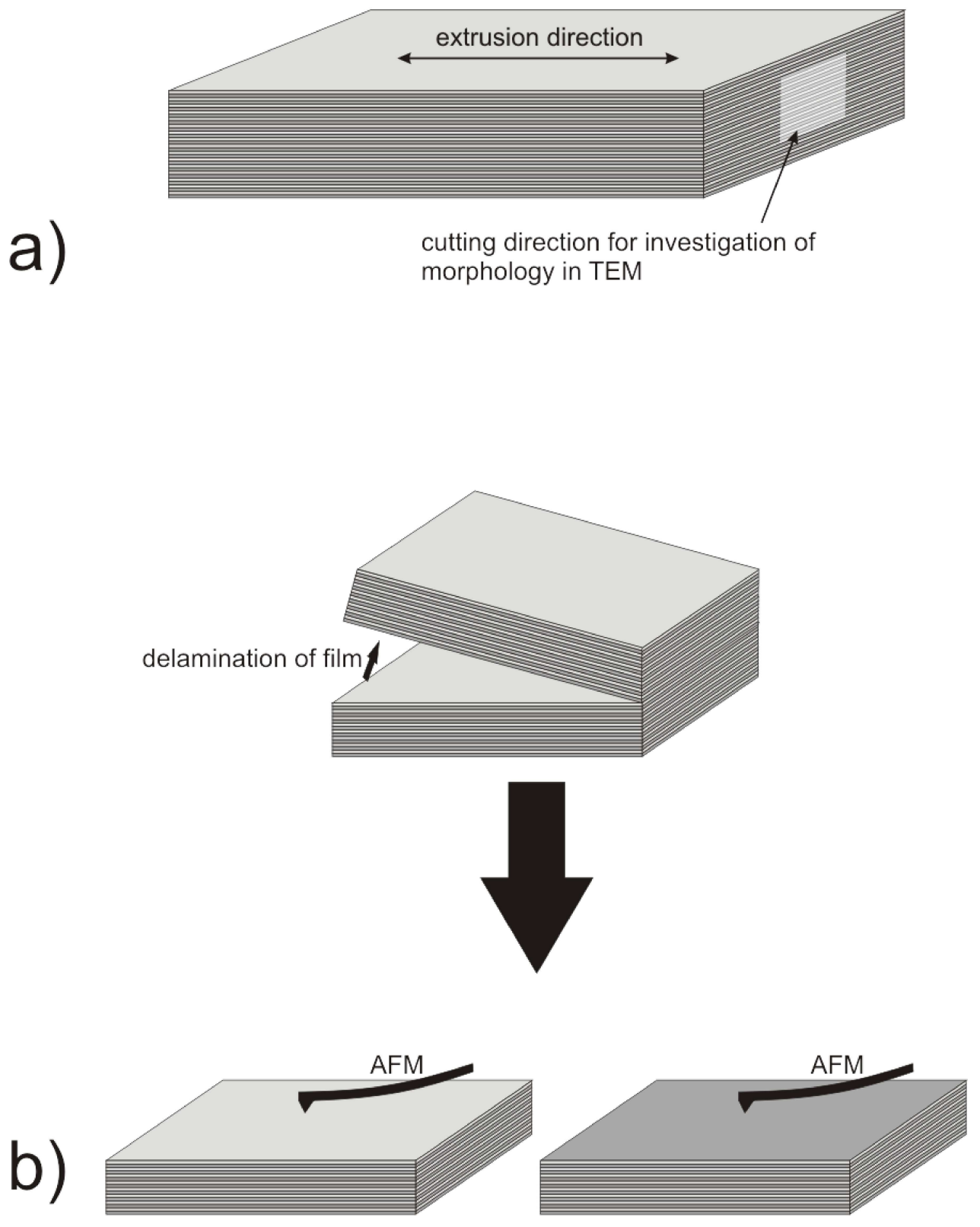

Fig. 1 


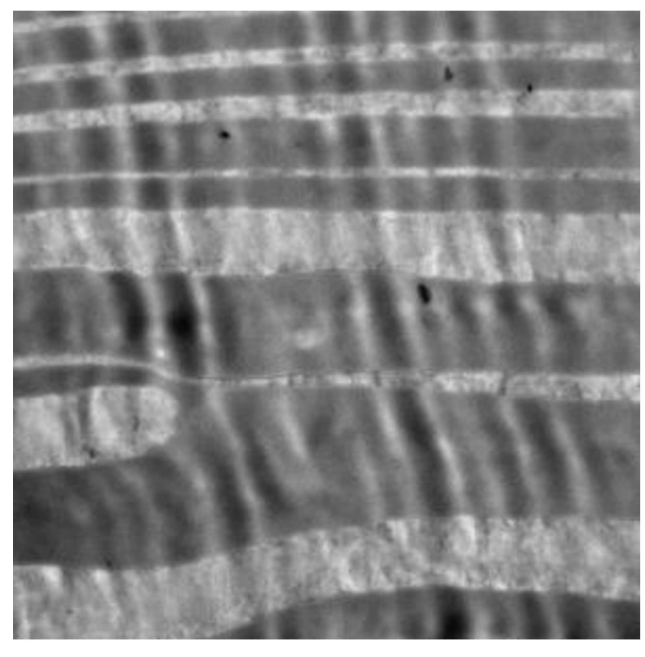

a

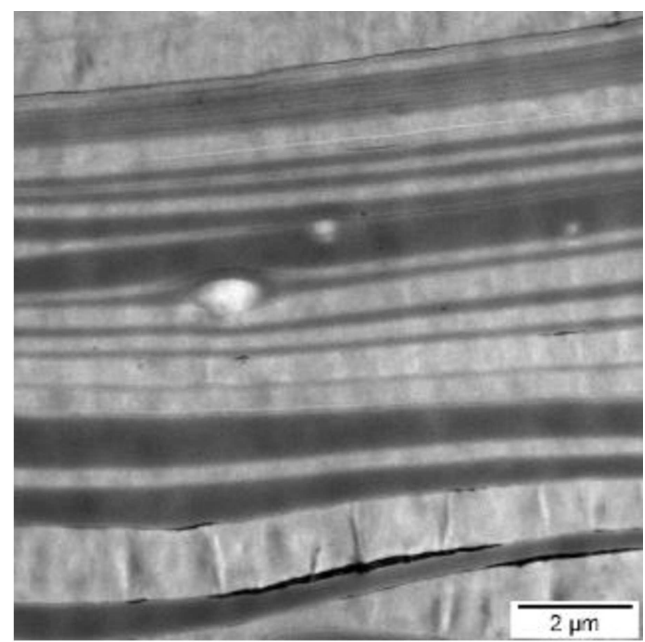

b

Fig. 2 


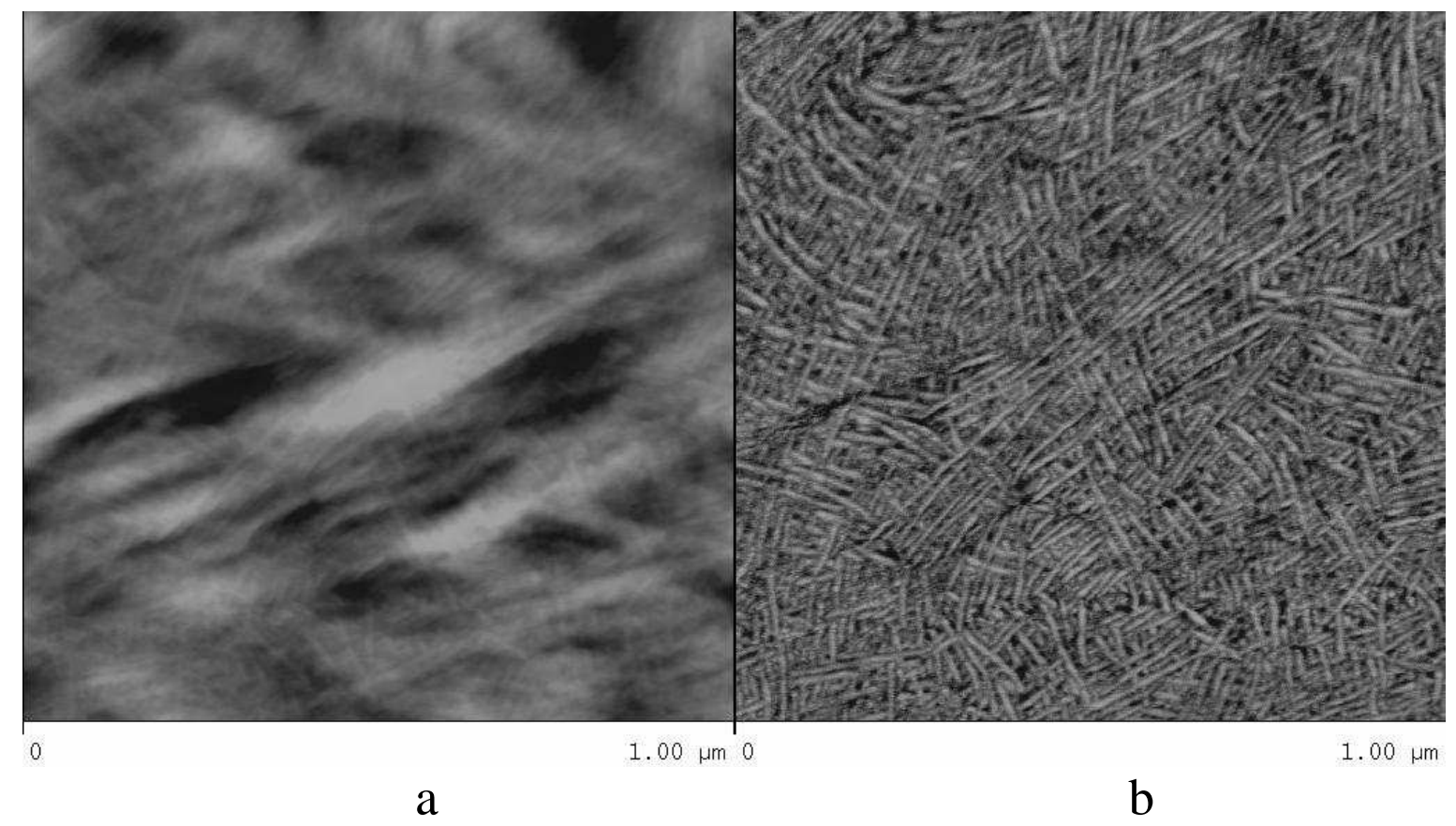

Fig. 3 


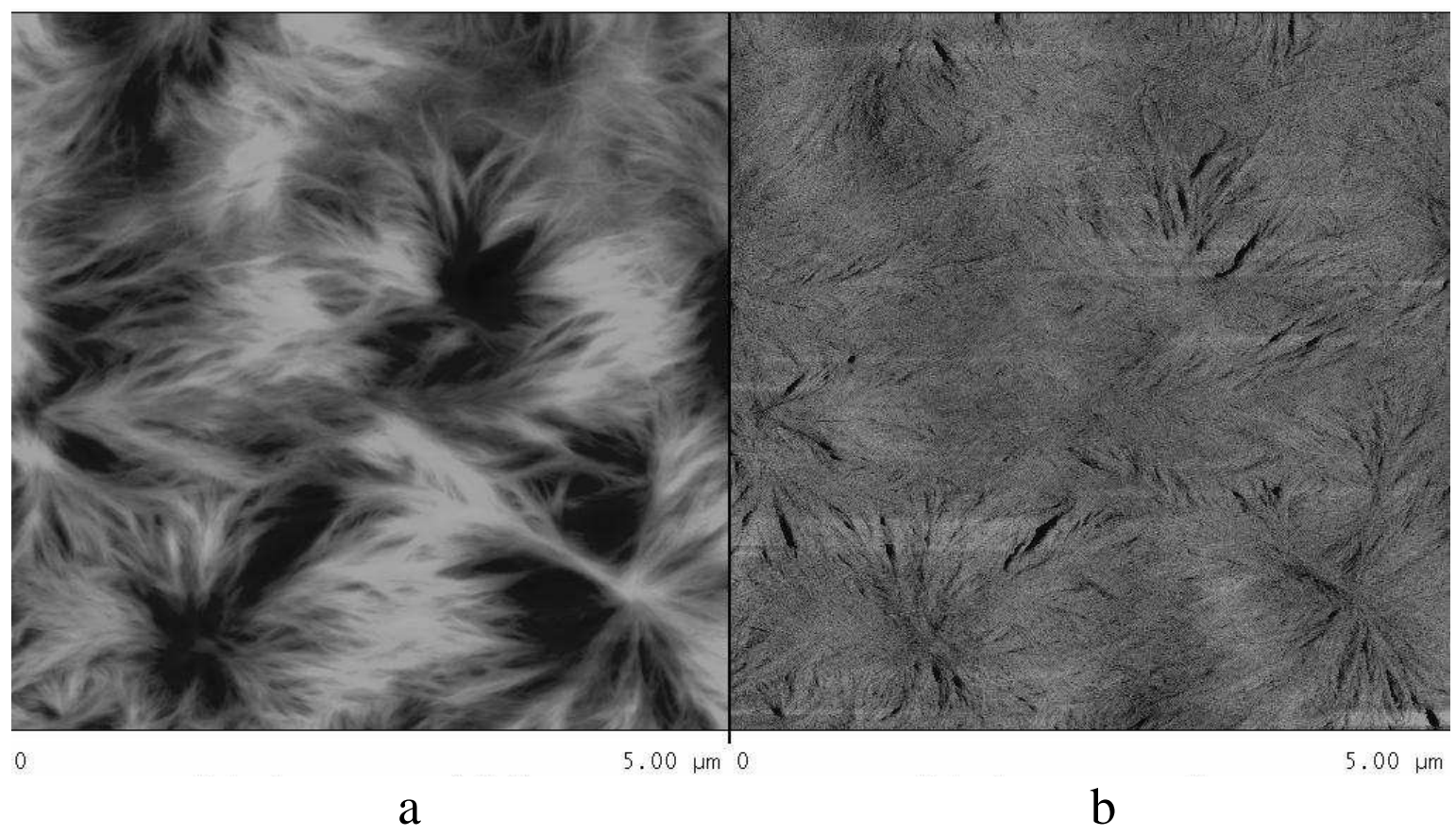

Fig.4 

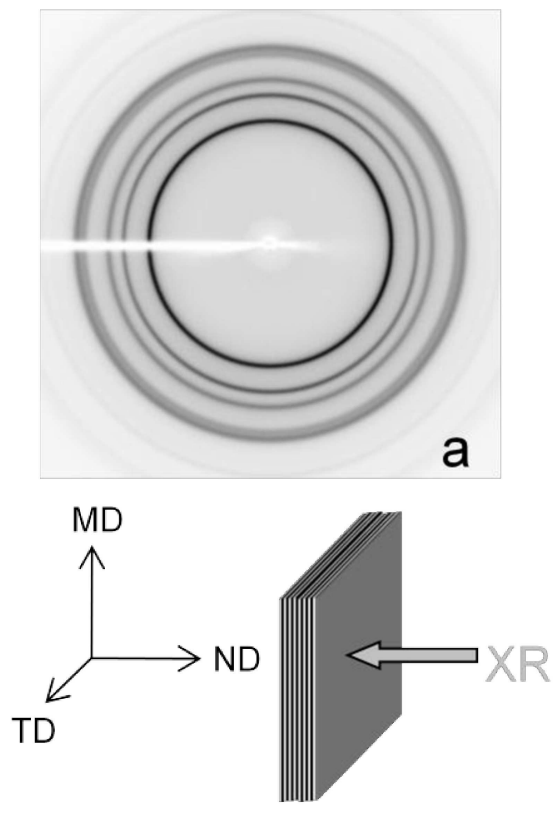

C
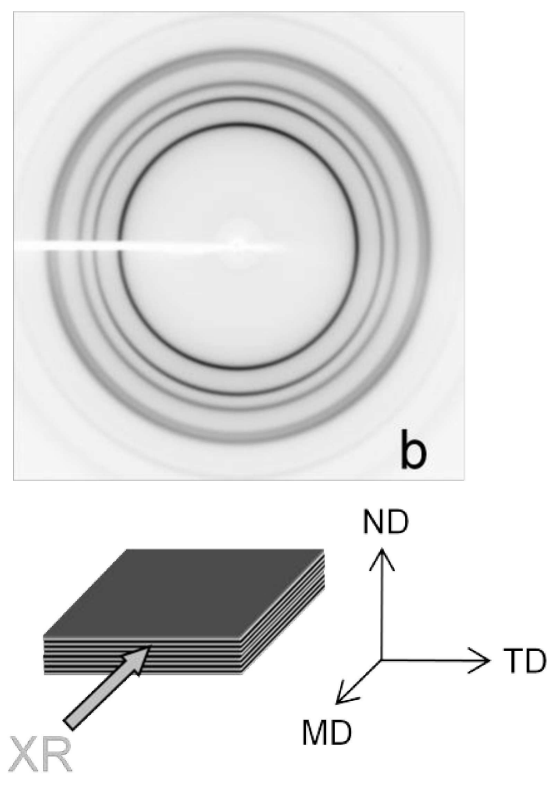

d

Fig. 5 
Fig. 6 

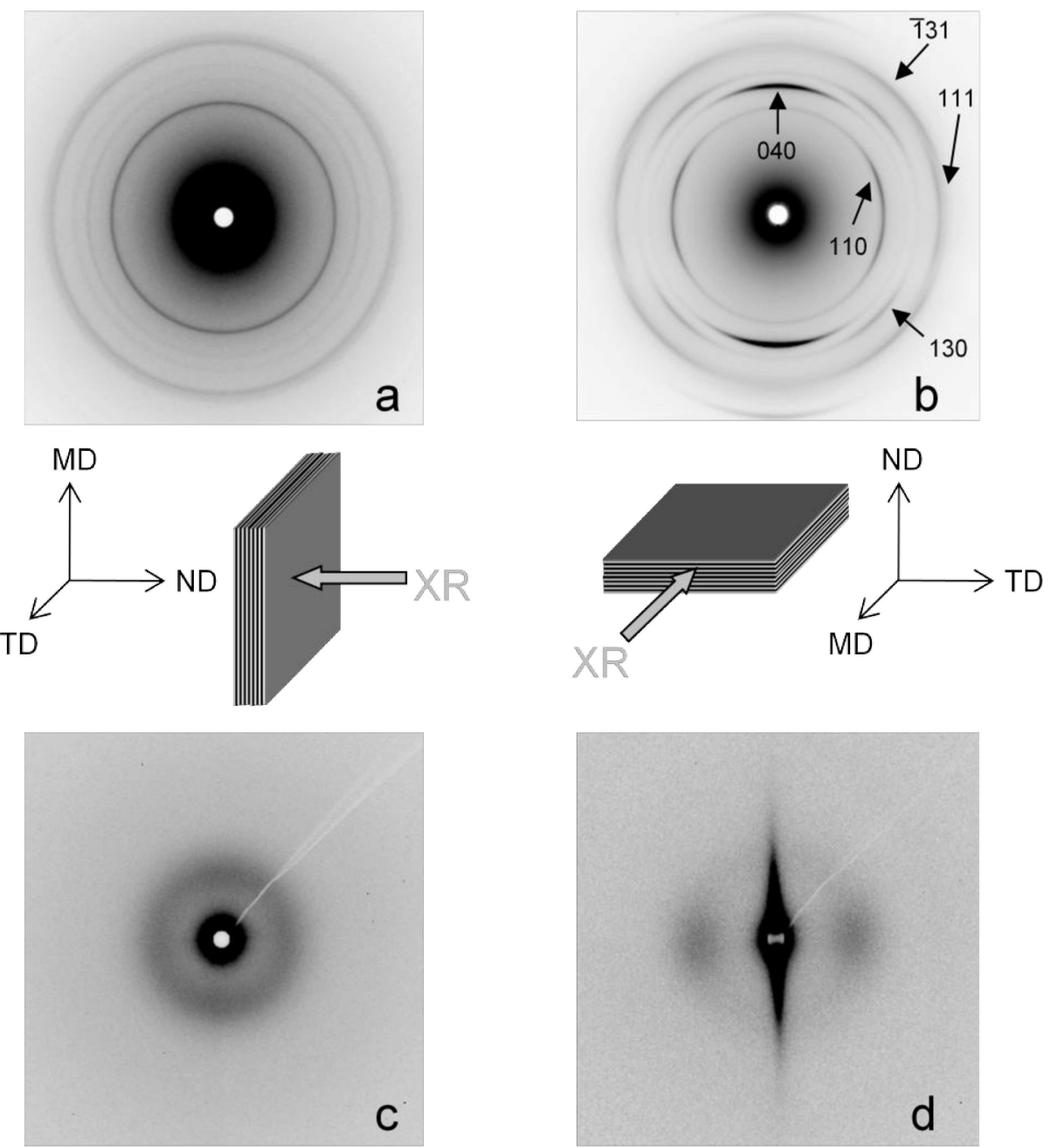

Fig. 7 

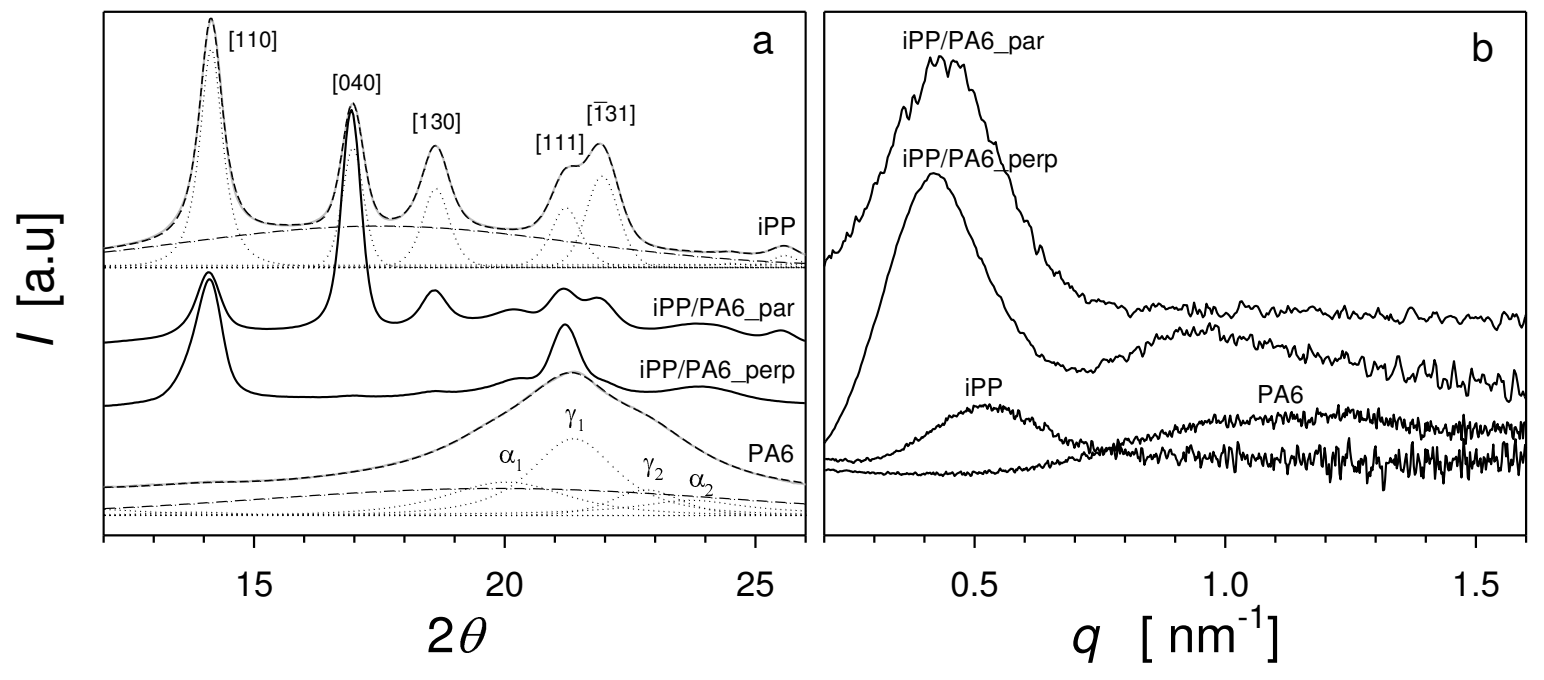

Fig. 8 


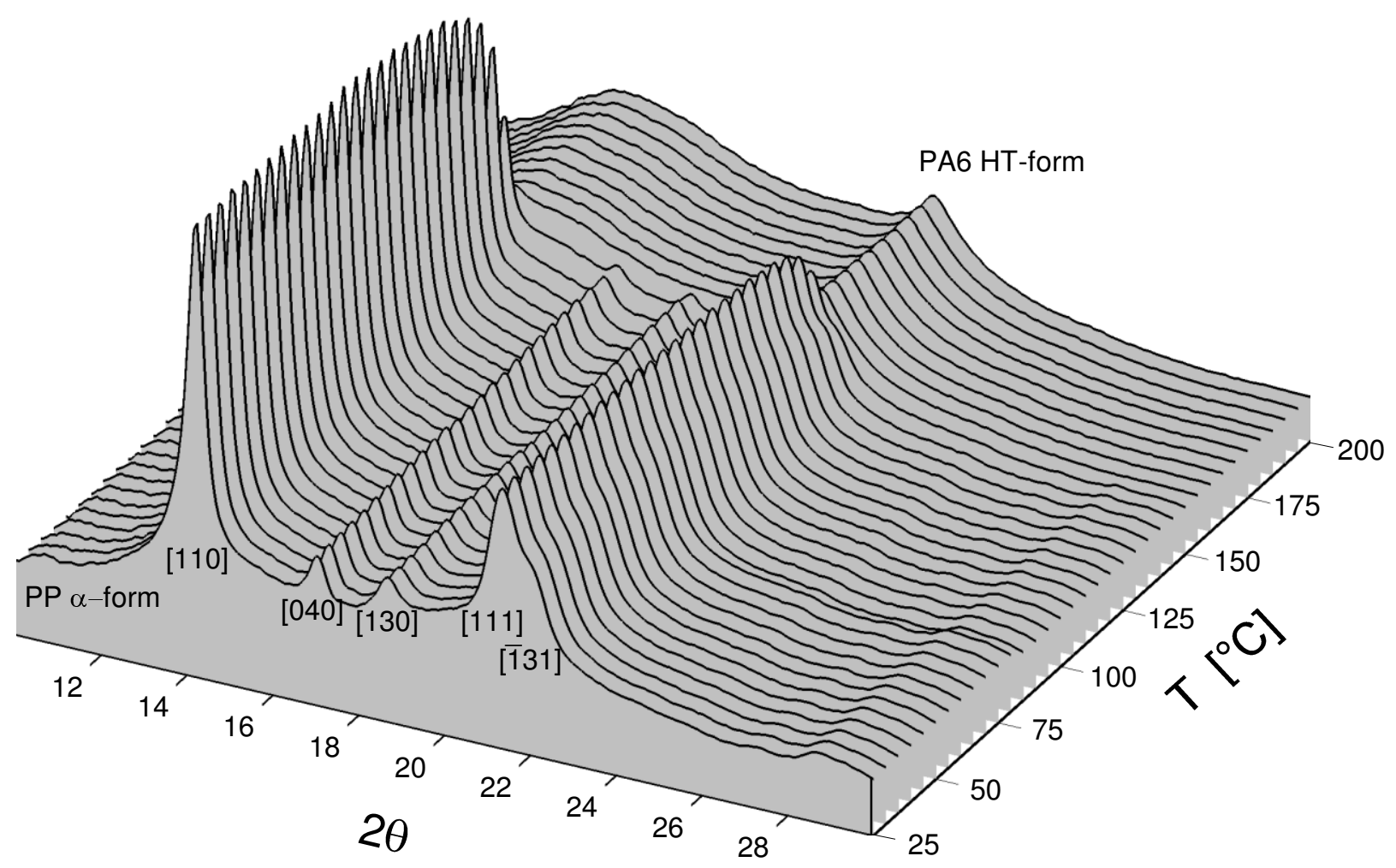

Fig. 9 


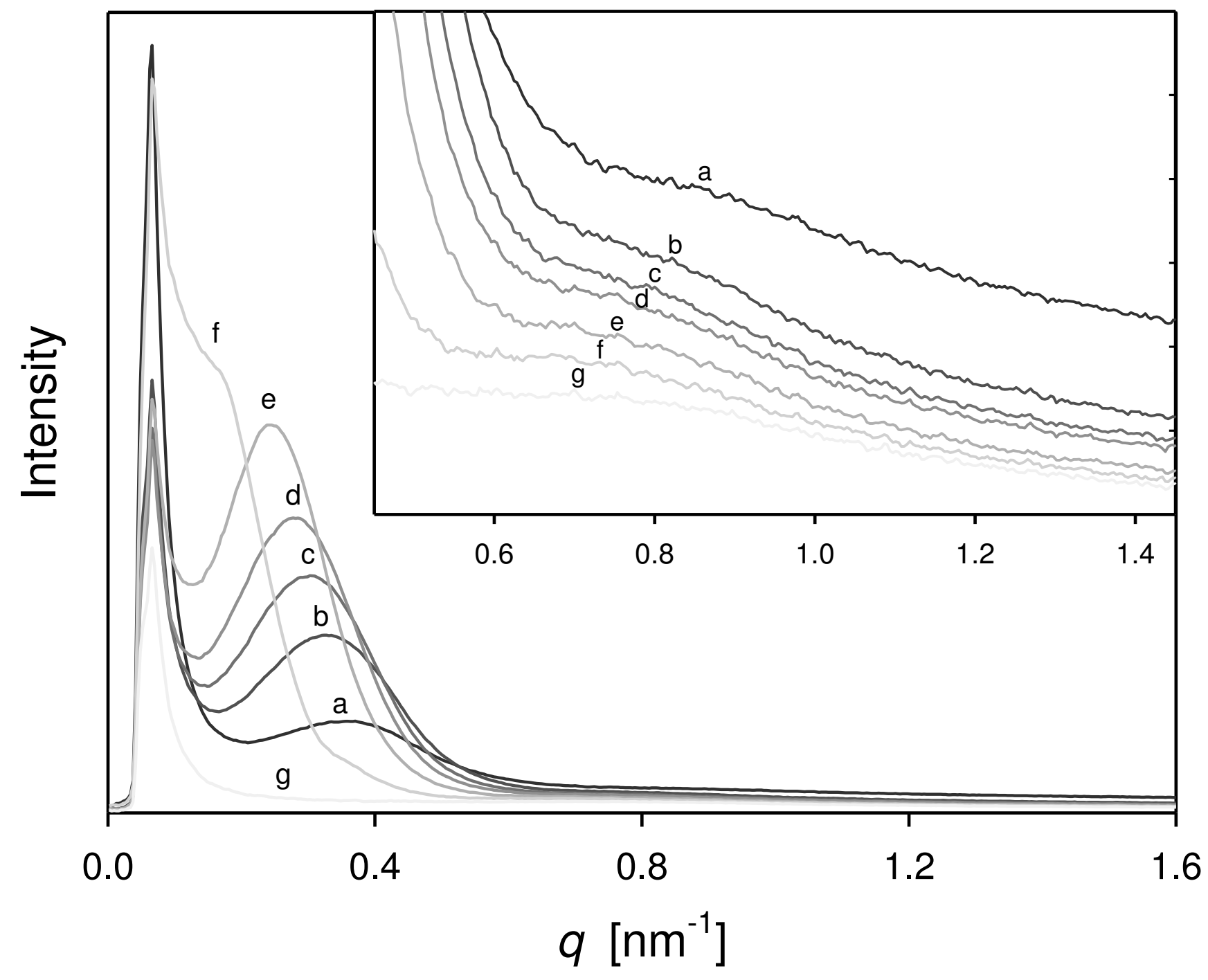

Fig. 10 


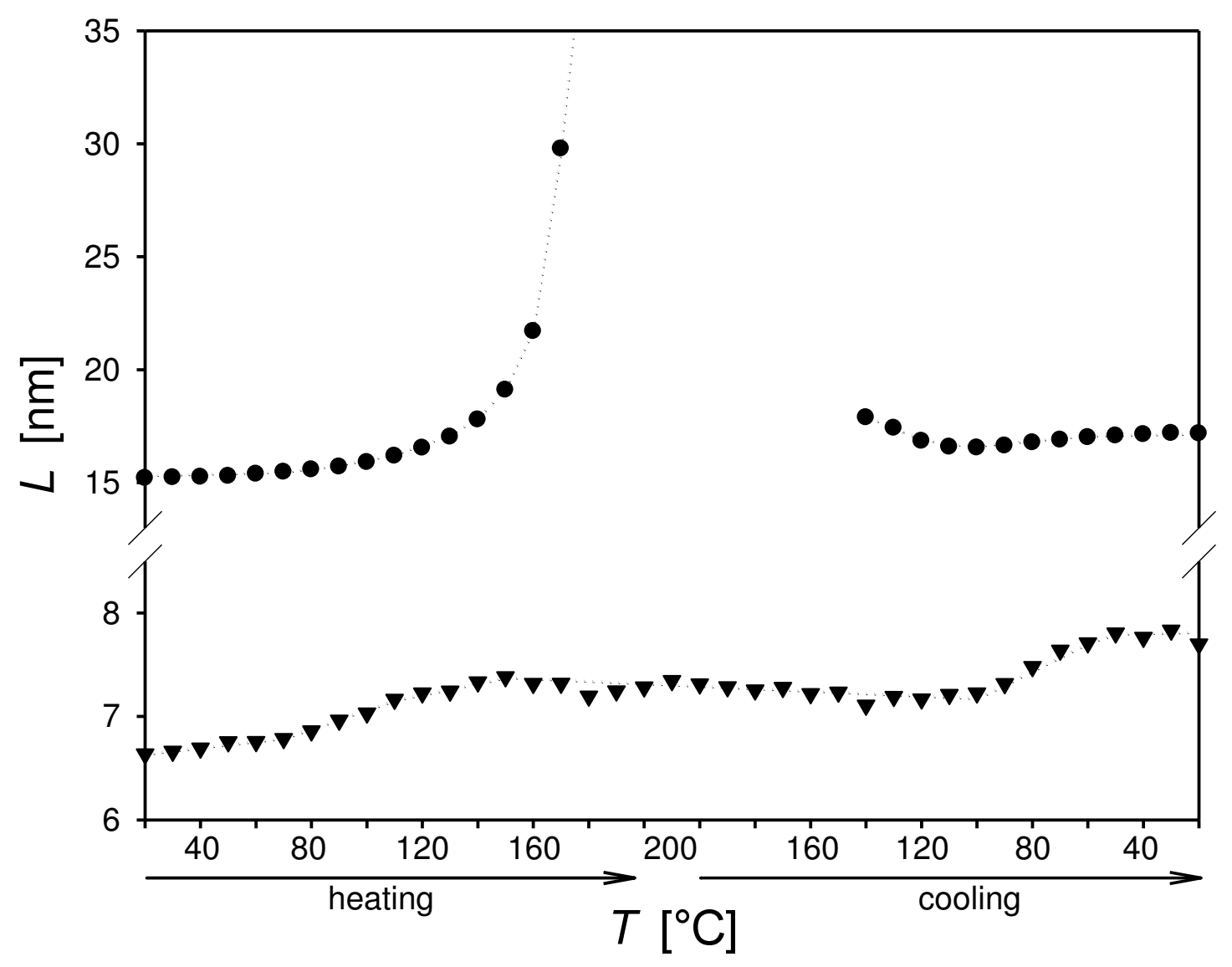

Fig. 11 